\title{
Loosening of the mesothelial barrier as an early therapeutic target to preserve peritoneal function in peritoneal dialysis
}

\author{
Duk-Hee Kang \\ Division of Nephrology, Department of Internal Medicine, Ewha Womans University School of Medicine, Ewha Medical Research \\ Center, Seoul, Republic of Korea
}

\begin{abstract}
Phenotype transition of peritoneal mesothelial cells (MCs) including the epithelial-to-mesenchymal transition (EMT) is regarded as an early mechanism of peritoneal dysfunction and fibrosis in peritoneal dialysis (PD), producing proinflammatory and pro-fibrotic milieu in the intra-peritoneal cavity. Loosening of intercellular tight adhesion between adjacent MCs as an initial process of EMT creates the environment where mesothelium and submesothelial tissue are more vulnerable to the composition of bio-incompatible dialysates, reactive oxygen species, and inflammatory cytokines. In addition, down-regulation of epithelial cell markers such as E-cadherin facilitates de novo acquisition of mesenchymal phenotypes in MCs and production of extracellular matrices. Major mechanisms underlying the EMT of MCs include induction of oxidative stress, pro-inflammatory cytokines, endoplasmic reticulum stress and activation of the local renin-angiotensin system. Another mechanism of peritoneal EMT is mitigation of intrinsic defense mechanisms such as the peritoneal antioxidant system and anti-fibrotic peptide production in the peritoneal cavity. In addition to use of less bio-incompatible dialysates and optimum treatment of peritonitis in PD, therapies to prevent or alleviate peritoneal EMT have demonstrated a favorable effect on peritoneal function and structure, suggesting that EMT can be an early interventional target to preserve peritoneal integrity.
\end{abstract}

Keywords: Adhesion molecule, Epithelial-to-mesenchymal transition, Peritoneal fibrosis, Peritoneal mesothelial cells

\section{Introduction}

An estimated 2 million people worldwide are affected

Received April 9, 2020; Revised May 8, 2020;

Accepted May 11, 2020

Edited by Gheun-Ho Kim, Hanyang University, Seoul, Republic of Korea

Correspondence: Duk-Hee Kang

Division of Nephrology, Ewha Womans University School of Medicine, 260 Gonghang-daero, Gangseo-gu, Seoul 07804, Republic of Korea. E-mail: dhkang@ewha.ac.kr

Copyright (c) 2020 by The Korean Society of Nephrology

(a) This is an open-access article distributed under the terms of the Creative Commons Attribution Non-Commercial License (http://creativecommons. org/licenses/by-nc-nd/4.0/), which permits unrestricted non-commercial use, distribution, and reproduction in any medium, provided the original work is properly cited. by end-stage renal disease (ESRD), and the number of patients newly diagnosed with ESRD continues to increase at a rate of $5 \%$ to $7 \%$ per year. Approximately $11 \%$ of ESRD patients receive peritoneal dialysis (PD) as renal replacement therapy, with an annual growth rate of $8 \%$ [1]. PD offers therapeutic advantages for appropriate patients, including early survival benefits, the convenience of home therapy, and lower healthcare costs in general $[2,3]$.

PD uses the patient's own peritoneal membrane instead of a disposable dialysis membrane in hemodialysis, across which waste products are exchanged from the circulation and flushed out through a peritoneal catheter. It is of paramount importance to maintain a healthy peritoneum. However, cumulative evidence based on basic and clinical studies has highlighted that continuous 
exposure to bio-incompatible compositions of peritoneal dialysate, the presence of catheter per se, recurrent episodes of peritonitis, and other factors lead to functional and structural damage of the peritoneal structure over time [4]. Peritoneal biopsy studies have demonstrated morphologic changes in long-term PD patients, which included loss of peritoneal mesothelial cells (MCs), accumulation of extracellular matrices (ECM), increased angiogenesis, and fibrosis $[5,6]$. Peritoneal fibrosis with extensive submesothelial thickening leads to a functional loss of the peritoneum as a semi-permeable dialysis membrane, ultimately resulting in discontinuation of PD.

Therefore, one of the most important challenges in the PD community is preservation of peritoneal membrane integrity. To improve the outcome of PD therapy and favorably expand its clinical application, there is a compelling need to understand the early mechanisms that trigger peritoneal membrane damage during PD. Previous studies have suggested the phenotype transition of peritoneal MCs before evident morphologic change becomes recognizable under light microscopy [7-9]. During the process of phenotype transition, there is disruption of the tight junction and acquisition of migratory and invasive phenotypes in MCs, followed by production of ECM [1012]. The phenotype transition of peritoneal MCs, such as epithelial-to-mesenchymal transition (EMT), is regarded as one of the earliest phenomena of peritoneal dysfunction in PD [7]. The molecular mechanism of EMT and its regulation are of interest as potential therapeutic targets to ameliorate peritoneal fibrosis $[12,13]$.

In this review, the evidence of EMT in peritoneal fibrosis and its mechanisms will be discussed with the proposal of therapeutic modalities targeting peritoneal EMT.

\section{Peritoneal MCs as the first line of defense and master regulator of peritoneal function}

Peritoneal MC cells are epithelial-like cells resting on a thin basement membrane that lines the entire abdominal cavity [14]. The MC monolayer ("mesothelium") is recognized as the first-line barrier that provides a protective, non-adhesive surface on the abdominal cavity and organs. Peritoneal MCs predominantly adopt a polygonal cobblestone-like morphology and are supported by submesothelial connective tissue containing blood vessels, lymphatics, and resident fibroblast-like cells [15].
The boundaries between MCs consist of delicate junctional complexes, including tight junctions, adherens junctions, gap junctions, and desmosomes, which are crucial for maintenance of a tight semi-permeable diffusion barrier [14]. In addition, MCs are not just a simple barrier, but play a critical role in regulating peritoneal homeostasis by secreting diverse mediators governing immune surveillance, tissue repair, angiogenesis, inflammatory responses, and control of fluid and solute transport [15-17]. Therefore, functional and structural alterations in MCs may trigger signals that lead to irreversible peritoneal damage.

\section{EMT of peritoneal MCs}

EMT is a biological process that allows an epithelial cell to acquire a mesenchymal cell phenotype through multiple biological processes resulting in enhanced migratory capacity, invasiveness, self-renewal properties, and increased production of ECM [18-20]. EMT is a physiologic process of organ development and wound healing through a finely programmed process. However, it can be pathologically continued in response to internal or external stimuli such as inflammation, eventually leading to organ fibrosis. EMT was once known as an irreversible process; however, several studies have revealed the reversible potential of peritoneal EMT [21]. During EMT, the earliest event involves loosening of cell-to-cell contacts between neighboring MCs [18], which is associated with downregulation of epithelial adherens junctions (like E-cadherin) and tight junction components (like zonula occludens-1, ZO-1). Tight junction proteins such as claudins and occludin are para-cellular components regulating cellular transport of peritoneal mesothelium. The expression and/or intracellular localization of tight junction proteins of MCs are altered by peritoneal dialysates in ESRD patients [22], which are mediated by induction of oxidative stress [23]. The alteration of adhesion and tight junction proteins can reflect an impairment of mesothelial integrity as a peritoneal barrier. Importantly, decrease of E-cadherin expression per se in MCs is known to upregulate the expression of mesenchymal junctional proteins (such as N-cadherin) and switch main cytoskeletal protein patterns of MCs from cytokeratin to vimentin with de novo synthesis of fibroblastspecific protein 1 and smooth muscle actin ( $\alpha$-SMA) 
[18-20,24]. It has been well demonstrated that EMT or mesothelial-to-mesenchymal transition of peritoneal MCs leads to accumulation of myofibroblasts that are distinct from peritoneal residential fibroblasts in phenotype. Loosening of intercellular tight adhesions also creates an environment that makes MCs and submesothelial tissue more vulnerable to the composition of bio-incompatible dialysates, reactive oxygen species (ROS), and inflammatory cytokines in PD patients (Fig. 1).

Despite recent controversy on the source of myofibroblasts in organ fibrosis, current evidence in animal PD models and patients supports the role of EMT as a key mechanism of the production of myofibroblast. In the seminal study by Yáñez-Mó et al [7], EMT was observed in MCs isolated from the effluent of PD patients even at the early time point of PD initiation, indicated by a progressive loss of epithelial morphology and a decrease in the expression of E-cadherin and cytokeratin. There are many direct/indirect findings supporting the presence of peritoneal EMT. Cultured MCs undergo transdifferentiation to mesenchymal cells upon exposure to various stimuli including high glucose, glucose degradation products (GDP), proinflammatory cytokines, and peritoneal dialysates. Furthermore, immunohistochemical analysis revealed the coexistence of epithelial and mesenchymal markers within MCs during peritoneal fibrosis [25-27]. Intraperitoneal transfection of transforming growth factor (TGF)- $\beta 1$ induced EMT and peritoneal fibrosis in rats, which was associated with disruption of an intact mesothelial layer and appearance of cytokeratin $+/ \alpha$-SMA+ spindle-shaped cells in the submesothelial compact zone [28]. Consistent with this animal model, a clinical study with 35 PD patients demonstrated in situ evidence of EMT (submesothelial cytokeratin staining) in parietal peritoneal tissue of $17 \%$ of patients and loss of the mesothelial layer in $74 \%$ of patients, indicating that conversion of epithelial-like MCs to fibroblast-like phenotypes was frequent in the peritoneal membrane during

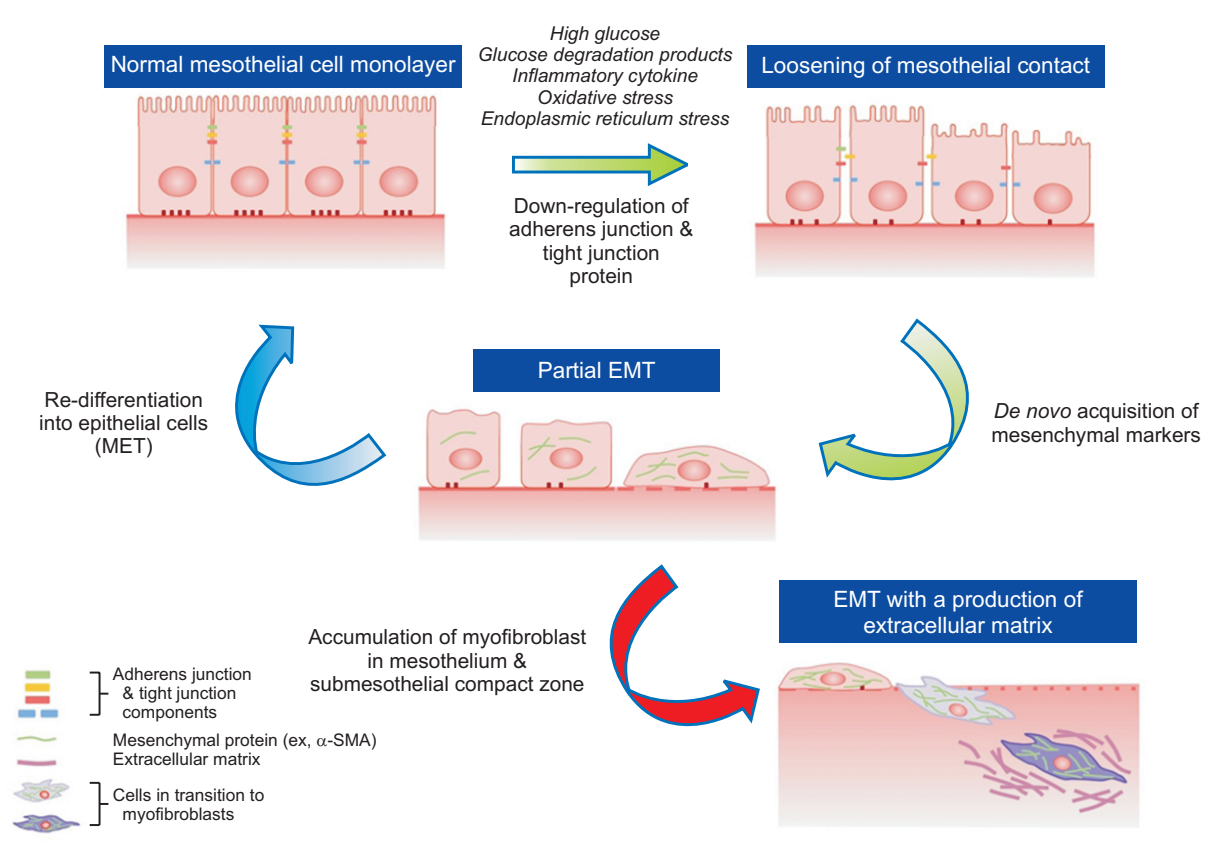

Figure 1. Epithelial-to-mesenchymal transition (EMT) as a key mechanism of peritoneal fibrosis. Transition of epithelial cells (peritoneal mesothelial cells [MCs]) toward a mesenchymal phenotype can be initiated by alteration of peritoneal milieu induced by the peritoneal dialysis process and related pro-inflammatory and pro-oxidative stress. Loss of cell-to-cell contact and cell polarity is one of the earliest phenomena of this continuous process and is associated with down-regulation of adhesion components between adjacent epithelial cells. Loosening of cell contacts per se leads to de novo acquisition of mesenchymal phenotypes. Cells in the process of transition before invasion beyond the basement membrane and migration into the submesothelial zone ("Partial EMT," green arrow) can be re-differentiated into MCs (mesenchymal-to-epithelial transition [MET], blue arrow) through either removal of EMT-inducing stimuli or reinforcement of the intraperitoneal defense mechanism. Instead of halting the process of phenotype transition of MCs, EMT may result in formation of myofibroblasts with production of extracellular matrices, which seems to be irreversible and results in peritoneal fibrosis.

SMA, smooth muscle actin. 
PD therapy [29]. A number of subsequent studies demonstrated similar changes in the peritoneal membrane [3033], suggesting local conversion of MCs to myofibroblasts after PD initiation.

\section{Mechanism of EMT}

Major mechanisms underlying peritoneal EMT include induction of oxidative stress, pro-inflammatory cytokines, endoplasmic reticulum (ER) stress and activation of the local renin-angiotensin system. Based on experimental data using TGF- $\beta 1$, the most potent pro-fibrotic cytokine known to induce EMT, the balance among Smad2, Smad3, and Smad7 plays a role in the development of EMT of MCs [34]. Smad proteins, phosphorylated by TGF- $\beta 1$ stimulation, control transcription of TGF- $\beta 1$ responsive genes [34,35], many of which are identified as pro-fibrogenic genes and regulators of EMT in peritoneal fibrosis, including Snail $[36,37]$, fibronectin $[38,39]$, connective tissue growth factor [40], $\beta$-catenin [41], monocyte chemoattractant protein-1 [42], and matrix metalloproteinase-2 $[43,44]$. Therefore, TGF- $\beta 1$-induced mesothelial transition leads to a characteristic myofibroblastic phenotype and complex modulation of gene expression, including cytoskeletal organization, cell adhesion, and ECM production $[8,45]$. There is also emerging evidence showing the role of non-Smad pathways in peritoneal EMT, which includes extracellular signalregulated kinase (ERK)/nuclear factor kappa B [46], mitogen-activated protein kinase (MAPK) [21], Akt/mTOR [9], c-Jun $\mathrm{N}$-terminal kinase $[47,48]$, and Wnt/ $\beta$-catenin [49]. TGF- $\beta 1$ was also reported to induce ER stress [50], activate NLRP3 inflammasomes [51], and inhibit the expression of $5^{\prime}$-adenosine monophosphate-activated protein kinase [27] in peritoneal MCs.

Among these complicated networks of molecular pathway, induction of oxidative stress is an early key mechanism of peritoneal EMT [52-54]. A major source of ROS in MCs is NADPH oxidase (NOX), mitochondrial electron transport, xanthine oxidase, and nitric oxide synthase. TGF $\beta 1$ increased NOX activity with induction of both membranous NOX- and mitochondria-mediated ROS production with as little as 15 minutes of TGF $\beta 1$ stimulation [48]. MCs possess the unique ROS production machinery of NOXl as the most abundant isoform and mitochondrial NOX4, which are differentially regulated in transcription, translation, and translocation of $\mathrm{p} 47 \mathrm{phox}$ into the cell membranes and enhancement of p47phoxp22phox binding [51]. Peritoneal dialysate or high glucose is reported to induce an alteration in the expression of tight junction proteins such as claudin-1, claudin-2, and occludin with increased permeability through induction of oxidative stress [55].

Another important mechanism of peritoneal EMT involves mitigation of intrinsic defense mechanisms such as the peritoneal antioxidant system and anti-fibrotic peptide production in the peritoneal cavity. In the animal model of PD, increased oxidative stress through alleviation of mesothelial antioxidant production is expressed as a decrease in the ratio of reduced to oxidized glutathione (GSH/GSSG) and superoxide dismutase (SOD) activity and an increase of 8-hydroxy deoxyguanosine in the peritoneal dialysate. Particularly, SOD activity in peritoneal effluent was shown to be almost absent in rats on PD [27]. In addition, peritoneal MCs are known to constitutively synthesize hepatocyte growth factor (HGF) and bone morphogenic peptide-7 (BMP-7), which are wellknown anti-fibrotic peptides. High glucose or TGF $\beta 1$ decreases the production of HGF and BMP-7, suggesting weakening of a natural anti-fibrotic mechanism of peritoneum by the process of PD [25].

\section{Potential therapies targeting peritoneal EMT}

The use of less bio-incompatible dialysates such as low-GDP or bicarbonate-based dialysate was reported to protect the peritoneum from EMT with better preservation of peritoneal morphology $[31,38]$. An alternative approach to maintaining the peritoneal MC barrier and preserving peritoneal integrity is therapy targeting EMT. Although the presence of peritoneal EMT and its mechanism have been extensively studied, there are not many studies showing the beneficial effect of therapy targeting peritoneal EMT per se. Table 1 shows the previous studies regarding EMT-targeting interventions using in vitro and in vivo experimental models [21,25-27,51,56-64].

The PPAR- $\gamma$ agonist rosiglitazone was reported to preserve the MC monolayer with reduction of peritoneal fibrosis and angiogenesis in mice models of PD [56]. Anti-oxidants, including $\mathrm{N}$-acetyl cysteine (NAC, ROS scavenger), apocynin (NOX inhibitor), and mitoQ (an inhibitor of mitochondrial electron transfer chain sub- 
Table 1. Therapies targeting peritoneal EMT

\begin{tabular}{|c|c|c|c|c|}
\hline References & Agent & Design & Model & Result \\
\hline Sandoval et al [56] & Rosiglitazone & In vivo & Female C57BL/6 mice & $\begin{array}{l}\text { Ameliorates PDS-induced EMT, } \\
\text { angiogenesis, and peritoneal fibrosis }\end{array}$ \\
\hline Jang et al [21] & Dexamethasone & In vitro & MCs isolated from omentum & Ameliorates TGF $\beta 1$-induced EMT \\
\hline Loureiro et al [57] & Tamoxifen & $\begin{array}{l}\text { In vitro and } \\
\text { in vivo }\end{array}$ & $\begin{array}{l}\text { MCs isolated from omentum/PD } \\
\text { effluent and female C57BL/6 } \\
\text { mice }\end{array}$ & $\begin{array}{l}\text { Blocks TGF } \beta 1 \text {-induced EMT and } \\
\text { decreases PDS-induced peritoneal } \\
\text { membrane thickness }\end{array}$ \\
\hline Liu et al [58] & Selenium & In vitro & HMrSV5 (human peritoneal MC line) & Inhibits LPS-induced EMT \\
\hline Yang et al [59] & $\begin{array}{l}\text { C646 (histone } \\
\text { acetyltransferase } \\
\text { inhibitor) }\end{array}$ & In vitro & HMrSV5(human peritoneal MC line) & Counteracts high glucose-induced EMT \\
\hline Shin et al [27] & $\begin{array}{l}\text { Metformin or AMPK } \\
\text { agonist }\end{array}$ & $\begin{array}{l}\text { In vitro and } \\
\text { in vivo }\end{array}$ & $\begin{array}{l}\text { MCs isolated from omentum } \\
\text { and Sprague-Dawley rats }\end{array}$ & $\begin{array}{l}\text { Ameliorates TGF } \beta 1 \text {-induced EMT } \\
\text { and PDS-induced EMT/peritoneal } \\
\text { thickening }\end{array}$ \\
\hline Ko et al [51] & Paricalcitol & In vitro & MCs isolated from omentum & Attenuates TGF $\beta 1$-induced EMT \\
\hline Ko et al [51] & $\begin{array}{l}\text { Blockers of NLRP3 } \\
\text { inflammasome }\end{array}$ & In vitro & MCs isolated from omentum & Attenuates TGF $\beta 1$-induced EMT \\
\hline Zhao et al [60] & Curcumin & In vitro & HMrSV5 (human peritoneal MC line) & Suppresses PDS-induced EMT \\
\hline Lupinacci et al [61] & Olive leaf extract & In vitro & MeT-5A (human MC line) & Inhibits TGF $\beta 1$-induced EMT \\
\hline Sun et al [62] & Smad-7 plasmid & In vivo & Sprague-Dawley rats & Inhibits PDS-induced peritoneal fibrosis \\
\hline Kang et al [63] & Tranilast & $\begin{array}{l}\text { In vitro and } \\
\text { in vivo }\end{array}$ & $\begin{array}{l}\text { MCs isolated from omentum } \\
\text { and Sprague-Dawley rats }\end{array}$ & $\begin{array}{l}\text { Attenuates TGF } \beta 1 \text {-induced EMT and } \\
\text { PDS-induced peritoneal fibrosis }\end{array}$ \\
\hline
\end{tabular}

BMP-7, bone morphogenic peptide-7; EMT, epithelial-to-mesenchymal transition; HGF, hepatocyte growth factor; LPS, lipopolysaccharide; MCs, mesothelial cells; PD, peritoneal dialysis; PDS, peritoneal dialysis solution; TGF, transforming growth factor.

unit I), were shown to alleviate TGF- $\beta 1$-induced EMT of MCs [51]. Taurine-conjugated ursodeoxycholic acid, an ER stress blocker, also ameliorated TGF- $\beta 1$-induced EMT in peritoneal MCs [50]. Interestingly, pre-treatment with tunicamycin or thapsigargin for 4 hours (ER stress preconditioning) also protected MCs from TGF- $\beta 1$-induced EMT, demonstrating the role of ER stress as an adaptive response to protect MCs from EMT. Given the consideration that peritoneal MCs isolated from PD patients displayed an increase in the marker of ER stress GRP78/94 and was correlated with degree of EMT [50], modulation of ER stress in MCs could serve as a novel approach to ameliorate peritoneal damage in PD patients. Previous studies have also demonstrated how glucocorticoids and mineralocorticoids play a role in peritoneal EMT $[21,26]$. Dexamethasone inhibited TGF- $\beta 1$-induced EMT and RU486, a glucocorticoid receptor antagonist, blocking the effect of dexamethasone on TGF- $\beta 1$-induced EMT. The beneficial effect of dexamethasone on TGF$\beta 1$-induced EMT was mediated through amelioration of ERK and p38 MAPK phosphorylation. Dexamethasone also inhibited glycogen synthase kinase- $3 \beta$ phosphorylation and Snail upregulation induced by TGF- $\beta 1$, which were ameliorated by inhibitors of MAPK. A recent study showed the beneficial effect of paricalcitol on the EMT of peritoneal MCs by ameliorating oxidative stress and inflammatory reactions [51]. Paricalcitol alleviated NOX activity by blocking the interaction of p47phox with $\mathrm{p} 22$ and inhibiting mitochondrial NOX4 mRNA expression and activity, resulting in an amelioration of TGF $\beta 1$-induced oxidative stress and activation of NLRP3 inflammasomes. Interestingly, blocking the expression and activity of 
NLRP3 inflammasomes in MCs also blocked the EMT of MCs [48]. In addition to the effort to alleviate peritoneal injury by extrinsic stress, enhancement of intrinsic antifibrotic defense in the peritoneal cavity has also been studied. HGF provided the dosage-dependent prevention of EMT and peritoneal fibrosis in animal PD models [25]. Both BMP-7 peptides and gene transfection with an adenoviral vector of BMP-7 also protected MCs from EMT. Furthermore, adenoviral BMP-7 transfection decreased peritoneal EMT and ameliorated peritoneal thickening in animal models of PD.

There are other pharmacologic agents such as cyclooxygenase-2 inhibitors $[65,66]$, renin-angiotensin blockades [67], and heparin [68] showing beneficial effects on peritoneal fibrosis and ultrafiltration in animal models of PD.

Although several studies on the effect of intraperitoneal or oral pharmacologic intervention on peritoneal function have been conducted in PD patients [13], no prospective clinical studies have verified the benefit of clinical application of these agents.

\section{Debates on EMT as a Mechanism of Organ Fibrosis}

Despite evidence of the presence of EMT in organ fibrosis including peritoneal fibrosis, there has been a major challenge in understanding EMT as a prerequisite for organ fibrosis. Since Iwano et al [69] first hypothesized EMT as the mechanism of renal fibrosis by the lineagetracing strategy using transgenic reporter mice and bone marrow transplants, abundant studies providing experimental and clinical evidence for EMT as a key player of organ fibrosis have been published [70-76]. However, the role of EMT in renal and hepatic fibrosis has been recently challenged based on compelling findings in new lineage-tracing studies. Kriz et al [77] summarized these findings in their review and pointed out several "flaws" in previous studies of EMT, with a clear point of view that "unequivocal evidence supporting EMT as an in vivo process in renal fibrosis is lacking". Similar questions have also been raised about the role of EMT in peritoneal fibrosis [78]. Therefore, it may be worth investigating other potential sources of peritoneal myofibroblasts [78]. Nonetheless, it is certain that peritoneal MCs undergo the process of phenotype transitions whether or not they take the appearance of myofibroblasts expressing both epithelial and mesenchymal cells.

\section{Future perspectives}

Given the consideration of the reversible nature of EMT, it seems to be an attractive target for preventing or even reversing peritoneal damage. Although there are debates about whether EMT is indispensable for appearance of myofibroblast and peritoneal fibrosis, the phenotype transition of MCs clearly produces pro-inflammatory and pro-fibrotic microenvironments with induction of oxidative and ER stress in the abdominal cavity. Loosening of intercellular tight adhesions, which precedes loss of MCs and production of ECM, seems to be one of the targets to be reversed if it can be detected as early as possible. In addition, further studies regarding intervention targeting of EMT will be necessary through stimulation of active re-differentiation of MCs in the middle of EMT processes into healthy MCs (mesenchymal-to-epithelial transition) as well as inhibition of EMT.

\section{Conflicts of interest}

The author has no conflicts of interest to declare.

\section{Funding}

This work was supported by a grant from the National Research Foundation of Korea (NRF) funded by the Korean government (MSIP) (NRF-2017R1A2B2005849, NRF2020R1A2C3007759).

\section{References}

[1] Jain AK, Blake P, Cordy P, Garg AX. Global trends in rates of peritoneal dialysis. J Am Soc Nephrol 2012;23:533-544.

[2] Bargman JM. Advances in peritoneal dialysis: a review. Semin Dial 2012;25:545-549.

[3] Davies SJ. Peritoneal dialysis--current status and future challenges. Nat Rev Nephrol 2013;9:399-408.

[4] Pletinck A, Vanholder R, Veys N, Van Biesen W. Protecting the peritoneal membrane: factors beyond peritoneal dialysis solutions. Nat Rev Nephrol 2012;8:542-550.

[5] Williams JD, Craig KJ, von Ruhland C, Topley N, Williams GT; Biopsy Registry Study Group. The natural course of peritoneal membrane biology during peritoneal dialysis. 
Kidney Int Suppl 2003;(88):S43-S49.

[6] Williams JD, Craig KJ, Topley N, et al.; Peritoneal Biopsy Study Group. Morphologic changes in the peritoneal membrane of patients with renal disease. J Am Soc Nephrol 2002;13:470-479.

[7] Yáñez-Mó M, Lara-Pezzi E, Selgas R, et al. Peritoneal dialysis and epithelial-to-mesenchymal transition of mesothelial cells. N Engl J Med 2003;348:403-413.

[8] Yang AH, Chen JY, Lin JK. Myofibroblastic conversion of mesothelial cells. Kidney Int 2003;63:1530-1539.

[9] Patel P, Sekiguchi Y, Oh KH, Patterson SE, Kolb MR, Margetts PJ. Smad3-dependent and -independent pathways are involved in peritoneal membrane injury. Kidney Int 2010; 77:319-328.

[10] Margetts PJ, Bonniaud P. Basic mechanisms and clinical implications of peritoneal fibrosis. Perit Dial Int 2003;23: 530-541.

[11] Aroeira LS, Aguilera A, Sánchez-Tomero JA, et al. Epithelial to mesenchymal transition and peritoneal membrane failure in peritoneal dialysis patients: pathologic significance and potential therapeutic interventions. J Am Soc Nephrol 2007;18:2004-2013.

[12] López-Cabrera M. Mesenchymal conversion of mesothelial cells is a key event in the pathophysiology of the peritoneum during peritoneal dialysis. Adv Med 2014;2014:473134.

[13] González-Mateo GT, Aroeira LS, López-Cabrera M, RuizOrtega M, Ortiz A, Selgas R. Pharmacological modulation of peritoneal injury induced by dialysis fluids: is it an option? Nephrol Dial Transplant 2012;27:478-481.

[14] Mutsaers SE, Wilkosz S. Structure and function of mesothelial cells. Cancer Treat Res 2007;134:1-19.

[15] Yung S, Chan TM. Mesothelial cells. Perit Dial Int 2007;27 Suppl 2:S110-S115.

[16] Devuyst O, Margetts PJ, Topley N. The pathophysiology of the peritoneal membrane. J Am Soc Nephrol 2010;21:10771085.

[17] Schilte MN, Celie JW, Wee PM, Beelen RH, van den Born J. Factors contributing to peritoneal tissue remodeling in peritoneal dialysis. Perit Dial Int 2009;29:605-617.

[18] Kalluri R, Neilson EG. Epithelial-mesenchymal transition and its implications for fibrosis. J Clin Invest 2003;112:17761784.

[19] Acloque H, Adams MS, Fishwick K, Bronner-Fraser M, Nieto MA. Epithelial-mesenchymal transitions: the importance of changing cell state in development and disease. $J$ Clin Invest 2009;119:1438-1449.
[20] Thiery JP, Acloque H, Huang RY, Nieto MA. Epithelialmesenchymal transitions in development and disease. Cell 2009;139:871-890.

[21] Jang YH, Shin HS, Sun Choi H, et al. Effects of dexamethasone on the TGF- $\beta 1$-induced epithelial-to-mesenchymal transition in human peritoneal mesothelial cells. Lab Invest 2013;93:194-206.

[22] Ito T, Yorioka N, Yamamoto M, Kataoka K, Yamakido M. Effect of glucose on intercellular junctions of cultured human peritoneal mesothelial cells. J Am Soc Nephrol 2000;11: 1969-1979.

[23] Reyes JL, Molina-Jijón E, Rodríguez-Muñoz R, BautistaGarcía P, Debray-García Y, Namorado Mdel C. Tight junction proteins and oxidative stress in heavy metals-induced nephrotoxicity. Biomed Res Int 2013;2013:730789.

[24] Nieto MA. Epithelial plasticity: a common theme in embryonic and cancer cells. Science 2013;342:1234850.

[25] Yu MA, Shin KS, Kim JH, et al. HGF and BMP-7 ameliorate high glucose-induced epithelial-to-mesenchymal transition of peritoneal mesothelium. J Am Soc Nephrol 2009;20: 567-581.

[26] Yu M, Shin HS, Lee HK, et al. Effect of aldosterone on epithelial-to-mesenchymal transition of human peritoneal mesothelial cells. Kidney Res Clin Pract 2015;34:83-92.

[27] Shin HS, Ko J, Kim DA, et al. Metformin ameliorates the phenotype transition of peritoneal mesothelial cells and peritoneal fibrosis via a modulation of oxidative stress. Sci Rep 2017;7:5690.

[28] Margetts PJ, Bonniaud P, Liu L, et al. Transient overexpression of TGF- $\{$ beta 1 induces epithelial mesenchymal transition in the rodent peritoneum. J Am Soc Nephrol 2005;16: 425-436.

[29] Del Peso G, Jiménez-Heffernan JA, Bajo MA, et al. Epithelial-to-mesenchymal transition of mesothelial cells is an early event during peritoneal dialysis and is associated with high peritoneal transport. Kidney Int Suppl 2008;(108):S26S33.

[30] Jiménez-Heffernan JA, Aguilera A, Aroeira LS, et al. Immunohistochemical characterization of fibroblast subpopulations in normal peritoneal tissue and in peritoneal dialysisinduced fibrosis. Virchows Arch 2004;444:247-256.

[31] Do JY, Kim YL, Park JW, et al. The effect of low glucose degradation product dialysis solution on epithelial-to-mesenchymal transition in continuous ambulatory peritoneal dialysis patients. Perit Dial Int 2005;25 Suppl 3:S22-S25.

[32] Aroeira LS, Aguilera A, Selgas R, et al. Mesenchymal con- 
version of mesothelial cells as a mechanism responsible for high solute transport rate in peritoneal dialysis: role of vascular endothelial growth factor. Am J Kidney Dis 2005;46: 938-948.

[33] Oh EJ, Ryu HM, Choi SY, et al. Impact of low glucose degradation product bicarbonate/lactate-buffered dialysis solution on the epithelial-mesenchymal transition of peritoneum. Am J Nephrol 2010;31:58-67.

[34] Lan HY, Chung AC. TGF- $\beta /$ Smad signaling in kidney disease. Semin Nephrol 2012;32:236-243.

[35] Samarakoon R, Overstreet JM, Higgins PJ. TGF- $\beta$ signaling in tissue fibrosis: redox controls, target genes and therapeutic opportunities. Cell Signal 2013;25:264-268.

[36] Zhou Q, Yang M, Lan H, Yu X. miR-30a negatively regulates TGF- $\beta 1$-induced epithelial-mesenchymal transition and peritoneal fibrosis by targeting Snail. Am J Pathol 2013;183: 808-819.

[37] Hirahara I, Ishibashi Y, Kaname S, Kusano E, Fujita T. Methylglyoxal induces peritoneal thickening by mesenchymallike mesothelial cells in rats. Nephrol Dial Transplant 2009; 24:437-447.

[38] Bajo MA, Pérez-Lozano ML, Albar-Vizcaino P, et al. LowGDP peritoneal dialysis fluid ('balance') has less impact in vitro and ex vivo on epithelial-to-mesenchymal transition (EMT) of mesothelial cells than a standard fluid. Nephrol Dial Transplant 2011;26:282-291.

[39] Lei P, Jiang Z, Zhu H, Li X, Su N, Yu X. Poly(ADP-ribose) polymerase- 1 in high glucose-induced epithelial-mesenchymal transition during peritoneal fibrosis. Int J Mol Med 2012;29:472-478.

[40] Li X, Liu H, Sun L, et al. MicroRNA-302c modulates peritoneal dialysis-associated fibrosis by targeting connective tissue growth factor. J Cell Mol Med 2019;23:2372-2383.

[41] Yu M, Shi J, Sheng M, et al. Astragalus inhibits epithelial-tomesenchymal transition of peritoneal mesothelial cells by down-regulating $\beta$-catenin. Cell Physiol Biochem 2018;51: 2794-2813.

[42] Lee SH, Kang HY, Kim KS, et al. The monocyte chemoattractant protein-1 (MCP-1)/CCR2 system is involved in peritoneal dialysis-related epithelial-mesenchymal transition of peritoneal mesothelial cells. Lab Invest 2012;92: 1698-1711.

[43] Ertilav M, Timur O, Hür E, et al. What does the dialysate level of matrix metalloproteinase 2 tell us? Adv Perit Dial 2011;27:6-10.

[44] Hao N, Chiou TT, Wu CH, et al. Longitudinal changes of
PAI-1, MMP-2, and VEGF in peritoneal effluents and their associations with peritoneal small-solute transfer rate in new peritoneal dialysis patients. Biomed Res Int 2019;2019: 2152584.

[45] Margetts PJ, Oh KH, Kolb M. Transforming growth factorbeta: importance in long-term peritoneal membrane changes. Perit Dial Int 2005;25 Suppl 3:S15-S17.

[46] Strippoli R, Benedicto I, Pérez Lozano ML, Cerezo A, López-Cabrera M, del Pozo MA. Epithelial-to-mesenchymal transition of peritoneal mesothelial cells is regulated by an ERK/NF-kappaB/Snaill pathway. Dis Model Mech 2008;1:264-274.

[47] Liu Q, Mao H, Nie J, et al. Transforming growth factor \{beta\}1 induces epithelial-mesenchymal transition by activating the JNK-Smad3 pathway in rat peritoneal mesothelial cells. Perit Dial Int 2008;28 Suppl 3:S88-S95.

[48] Liu Q, Zhang Y, Mao H, et al. A crosstalk between the Smad and JNK signaling in the TGF- $\beta$-induced epithelial-mesenchymal transition in rat peritoneal mesothelial cells. PLoS One 2012; 7:e32009.

[49] Guo Y, Sun L, Xiao L, et al. Aberrant Wnt/beta-catenin pathway activation in dialysate-induced peritoneal fibrosis. Front Pharmacol 2017;8:774.

[50] Shin HS, Ryu ES, Oh ES, Kang DH. Endoplasmic reticulum stress as a novel target to ameliorate epithelial-to-mesenchymal transition and apoptosis of human peritoneal mesothelial cells. Lab Invest 2015;95:1157-1173.

[51] Ko J, Kang HJ, Kim DA, et al. Paricalcitol attenuates TGF$\beta 1$-induced phenotype transition of human peritoneal mesothelial cells (HPMCs) via modulation of oxidative stress and NLRP3 inflammasome. FASEB J 2019;33:3035-3050.

[52] Li Y, Wang L, Pappan L, Galliher-Beckley A, Shi J. IL-1 $\beta$ promotes stemness and invasiveness of colon cancer cells through Zeb1 activation. Mol Cancer 2012;11:87.

[53] Liang $\mathrm{H}$, Xu F, Zhang T, et al. Inhibition of IL-18 reduces renal fibrosis after ischemia-reperfusion. Biomed Pharmacother 2018;106:879-889.

[54] Guo J, Gu N, Chen J, et al. Neutralization of interleukin-1 beta attenuates silica-induced lung inflammation and fibrosis in C57BL/6 mice. Arch Toxicol 2013;87:1963-1973.

[55] Retana C, Sanchez E, Perez-Lopez A, et al. Alterations of intercellular junctions in peritoneal mesothelial cells from patients undergoing dialysis: effect of retinoic acid. Perit Dial Int 2015;35:275-287.

[56] Sandoval P, Loureiro J, González-Mateo G, et al. PPAR- $\gamma$ agonist rosiglitazone protects peritoneal membrane from 
dialysis fluid-induced damage. Lab Invest 2010;90:15171532.

[57] Loureiro J, Sandoval P, del Peso G, et al. Tamoxifen ameliorates peritoneal membrane damage by blocking mesothelial to mesenchymal transition in peritoneal dialysis. PLoS One 2013;8:e61165.

[58] Liu J, Zeng L, Zhao Y, Zhu B, Ren W, Wu C. Selenium suppresses lipopolysaccharide-induced fibrosis in peritoneal mesothelial cells through inhibition of epithelial-to-mesenchymal transition. Biol Trace Elem Res 2014;161:202-209.

[59] Yang Y, Liu K, Liang Y, Chen Y, Chen Y, Gong Y. Histone acetyltransferase inhibitor $\mathrm{C} 646$ reverses epithelial to mesenchymal transition of human peritoneal mesothelial cells via blocking TGF- $\beta 1 /$ Smad3 signaling pathway in vitro. Int $J$ Clin Exp Pathol 2015;8:2746-2754.

[60] Zhao JL, Guo MZ, Zhu JJ, Zhang T, Min DY. Curcumin suppresses epithelial-to-mesenchymal transition of peritoneal mesothelial cells (HMrSV5) through regulation of transforming growth factor-activated kinase 1 (TAK1). Cell Mol Biol Lett 2019;24:32.

[61] Lupinacci S, Perri A, Toteda G, et al. Olive leaf extract counteracts epithelial to mesenchymal transition process induced by peritoneal dialysis, through the inhibition of TGF $\beta 1$ signaling. Cell Biol Toxicol 2019;35:95-109.

[62] Sun Y, Zhu F, Yu X, et al. Treatment of established peritoneal fibrosis by gene transfer of Smad7 in a rat model of peritoneal dialysis. Am J Nephrol 2009;30:84-94.

[63] Kang SH, Kim SW, Kim KJ, et al. Effects of tranilast on the epithelial-to-mesenchymal transition in peritoneal mesothelial cells. Kidney Res Clin Pract 2019;38:472-480.

[64] Cheng S, Lu Y, Li Y, Gao L, Shen H, Song K. Hydrogen sulfide inhibits epithelial-mesenchymal transition in peritoneal mesothelial cells. Sci Rep 2018;8:5863.

[65] Aroeira LS, Lara-Pezzi E, Loureiro J, et al. Cyclooxygenase-2 mediates dialysate-induced alterations of the peritoneal membrane. J Am Soc Nephrol 2009;20:582-592.

[66] Fabbrini P, Schilte MN, Zareie M, et al. Celecoxib treatment reduces peritoneal fibrosis and angiogenesis and prevents ultrafiltration failure in experimental peritoneal dialysis. Nephrol Dial Transplant 2009;24:3669-3676.

[67] Duman S, Günal AI, Sen S, et al. Does enalapril prevent peritoneal fibrosis induced by hypertonic (3.86\%) peritoneal dialysis solution? Perit Dial Int 2001;21:219-224.

[68] De Vriese AS, Mortier S, Cornelissen M, et al. The effects of heparin administration in an animal model of chronic peritoneal dialysate exposure. Perit Dial Int 2002;22:566-572.

[69] Iwano M, Plieth D, Danoff TM, Xue C, Okada H, Neilson EG. Evidence that fibroblasts derive from epithelium during tissue fibrosis. J Clin Invest 2002;110:341-350.

[70] Carew RM, Wang B, Kantharidis P. The role of EMT in renal fibrosis. Cell Tissue Res 2012;347:103-116.

[71] Hills CE, Squires PE. The role of TGF- $\beta$ and epithelial-to mesenchymal transition in diabetic nephropathy. Cytokine Growth Factor Rev 2011;22:131-139.

[72] Fragiadaki M, Mason RM. Epithelial-mesenchymal transition in renal fibrosis - evidence for and against. Int $J$ Exp Pathol 2011;92:143-150.

[73] Quaggin SE, Kapus A. Scar wars: mapping the fate of epithelial-mesenchymal-myofibroblast transition. Kidney Int 2011;80:41-50

[74] Liu Y. New insights into epithelial-mesenchymal transition in kidney fibrosis. J Am Soc Nephrol 2010;21:212-222.

[75] Lee DB, Huang E, Ward HJ. Tight junction biology and kidney dysfunction. Am J Physiol Renal Physiol 2006;290:F20F34.

[76] Roberts AB, Tian F, Byfield SD, et al. Smad3 is key to TGFbeta-mediated epithelial-to-mesenchymal transition, fibrosis, tumor suppression and metastasis. Cytokine Growth Factor Rev 2006;17:19-27.

[77] Kriz W, Kaissling B, Le Hir M. Epithelial-mesenchymal transition (EMT) in kidney fibrosis: fact or fantasy? J Clin Invest 2011;121:468-474.

[78] Chen YT, Chang YT, Pan SY, et al. Lineage tracing reveals distinctive fates for mesothelial cells and submesothelial fibroblasts during peritoneal injury. J Am Soc Nephrol 2014; 25:2847-2858. 